\title{
Analysis of unsteady Lagrangian and Eulerian characteristics of a liquid fluidized bed by Direct Numerical Simulation.
}

\author{
Stéphane Vincent ${ }^{\dagger, a}$, Jean-Luc Estivalezes ${ }^{\uparrow}, *$, \\ Jorge César Brändle de Motta ${ }^{\boldsymbol{A}, *}$, Olivier Simonin*, \\ Olivier Masbernat ${ }^{o}$ \\ avincent@enscbp.fr (corresponding author)
}

† Université de Bordeaux, Institut de Mécanique et Ingénierie (I2M) -

UMR 5295, F-33400 Talence, France

- ONERA, The French Aerospace Lab, 2, avenue Edouard Belin, 31055

Toulouse, France

* Institut de Mécanique des Fluides de Toulouse IMFT, UMR 5502, Allée

Camille Soula, 31400 Toulouse, France

${ }^{\circ}$ Laboratoire de Génie chimique LGC, UMR 5503, Campus

INP-ENSIACET, 4 allée Emile Monso, 31432 Toulouse cedex 4, France

\begin{abstract}
The characterization of fluidized beds is still a challenging task for macroscopic modeling issues and industrial applications. The macroscopic models require to be fed with parameters or laws that are not well understood or even impossible to estimate as soon as the solid fraction is larger than 0.1 . The aim of the present work is to investigate Direct Numerical Simulation [1] of unsteady particle flows in order to solve all the time and space scale of the flow and the particle and to allow for the estimate of unknown macroscopic or stochastic characteristics of the flow. In the DNS, the particles are fully resolved, i.e. the particle diameter is larger than the grid size and to the smallest hydrodynamic scale. A benchmark experimental fluidized bed [2] is simulated and analyzed in terms of macroscopic and Lagrangian characteristics. Comparisons of numerical solutions to measurements are achieved with success.
\end{abstract}

keywords: DNS of fluidized bed, resolved scale particles, viscous penalty method 


\section{Introduction}

The characterization of dense liquid-solid flows and in particular fluidized beds is still a challenging task for macroscopic modeling issues and industrial applications as the granular dynamics based models or scale separation Eulerian-Eulerian or Eulerian-Lagrangian approaches. These macroscopic models require to be fed with parameters or laws that are not well understood or even impossible to estimate as soon as the solid fraction is larger than 0.1. The aim of the present work is to investigate Direct Numerical Simulation [1] of unsteady particle flows in order to solve all the time and space scale of the flow and the particle and to allow for the estimate of unknown macroscopic or stochastic characteristics of the flow. In the DNS, the particles are fully resolved, i.e. the particle diameter is larger than the grid size and to the smallest hydrodynamic scale.

Simulating a fluidized bed where particle and fluid have the same characteristic scales, i.e. resolved scale particle flows, is the scope of the present work. The Direct Point Simulation (DPS) approaches based on a Lagrangian modeling of the particles does not remain valid for that purpose [3] [4] [5]. The field of numerical methods devoted to the simulation of particulate flows involving finite size particles was widely developed the last 20 years concerning the study of the flow over a small number of fixed or moving particle [6] [7] [8] [9] [10] [11] or a fixed arrangement of spheres [12]. All the previously mentioned works were developed on fixed grids. The unstructured Arbitrary Eulerian-Lagrangian (ALE) grid simulations were developed for particle flows in the $90^{\prime} s$ by $\mathrm{Hu}$ et al. [6] concerning two-dimensional flows involving two particles. The works of Maury [13] with the ALE method are also to be noticed concerning the flow of 1000 non spherical particles in a two-dimensional biperiodic domain. A majority of the particulate flow simulations were carried out on structured grids to avoid the complexity of managing an evolving adapted mesh. Among them, the most relevant existing works concern the distributed Lagrangian multiplier (DLM) method of Glowinski and co-workers [14], the Physalis CFD code of Zhang and Prosperetti [15], the Immersed Boundary (IB) with direct forcing approach proposed by Uhlmann [16] [17], the lattice Boltzmann scheme [18, 19], the modified version of Uhlmann IB method published by Lucci et al. [20] [21] and the viscous penalty techniques of Ritz and Caltagirone [22] and Randrianarivelo et al. [23] [24] [1]. The present work uses the second order in space penalty method for the DNS of particulate flows based on the Implicit Tensorial Penalty Method [1]. 


\section{Simulation of real fluidized bed}

\subsection{Presentation of the fluidized bed}

The fluidized bed chosen for the simulations is taken from the experiments of Aguilar Corona [2]. In a cylinder of $0.64 m$ height and $0.08 m$ diameter, 2133 pyrex particles are fluidized by the circulation of potassium thiocyanate KSCN at various fluidization velocities. The diameter of the particles is $0.006 \mathrm{~m}$ and the simulated fluidization velocity is $0.12 \mathrm{~m} . \mathrm{s}^{-1}$. The density of the pyrex is $\rho_{s}=2230 \mathrm{~kg} \cdot \mathrm{m}^{-3}$ whereas the density $\rho_{l}$ and the dynamic viscosity $\mu_{l}$ of the KSCN are $1400 \mathrm{~kg} . \mathrm{m}^{-3}$ and $3.810^{-3}$ Pa.s. For the chosen particles in KSCN, the terminal settling velocity is equal to $0.226 \mathrm{~m} . \mathrm{s}^{-1}$, the particle Reynolds number based on this velocity is equal to 530 and the Stokes number is 0.8. Concerning the DNS of particles, a parallelepiped box of dimensions $8 \mathrm{~cm} \times 8 \mathrm{~cm} \times 64 \mathrm{~cm}$ is used with a grid mesh containing $160 \times 160 \times 1280$ cells. With such a grid resolution, nearly 12 cell length $\Delta x$ per particle diameter is achieved. The cylinder is penalized by a Darcy technique [25].

\subsection{Macroscopic results}

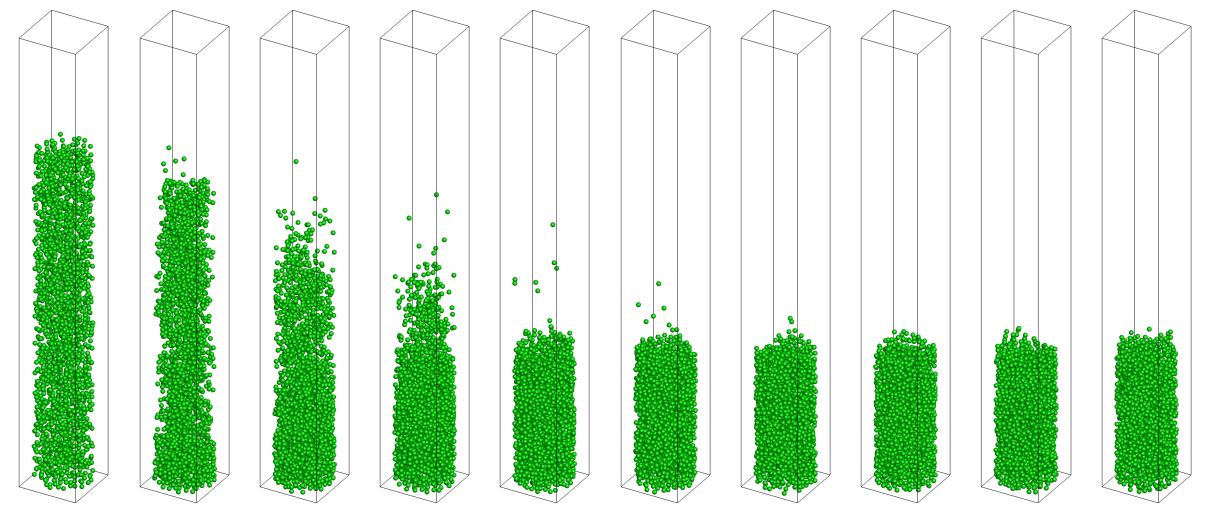

Figure 1: Three-dimensional view of the particles inside the fluidized bed from left to right, $t=1,2,3,4,5,6,7,8,9$ and $10 s$.

The macroscopic characteristics of the fluidized bed are first presented in order to validate the global interaction between the 2133 particles and the carrier fluid. The typical particle structures are illustrated in figure 1 over time. Is is observed that from $t=0 s$ to $6 s$, a packing occurs as the initial particle concentration $\alpha, i$. e. $\alpha=0.1$, is small compared to the fluidization velocity of $0.12 \mathrm{~m} . \mathrm{s}^{-1}$. After $6 s$, the fluidization regime is reached and the average bed height and resulting solid concentration in the vertical cylinder remain almost constant over time. The average numerical solid 
concentration $\alpha_{n}$ so obtained is 0.24 . For the chosen operating conditions, this value of $\alpha_{n}$ can be compared to the reference experimental correlation of Richardson and Zaki [26] which reads

$$
\alpha_{R Z}=1-\left(\frac{U_{0}}{U_{t}}\right)^{1 / 2.41}
$$

where the parameter 2.41 is correct for Reynolds numbers $R e_{t}$ based on the settling particle velocity larger than 500. In our configuration, $R e_{t}=530$. Concerning the fluidization velocity $U_{0}$ and the settling particle velocity $U_{t}$, the values of $0.12 \mathrm{~m}_{\mathrm{s}} \mathrm{s}^{-1}$ and $0.226 \mathrm{~m} . \mathrm{s}^{-1}$ are measured in the experiments of [2]. Finally, it is found that $\alpha_{R Z}=0.231$. A very good agreement is found between experiments and numerical solutions. This comparison validates the use of two numerical radii for the particles, one for the definition of the density and the treatment of the collisions and the other for the penalty viscosity. A correct global equilibrium between the gravity and drag forces is so obtained.

\subsection{Lagrangian characteristics}

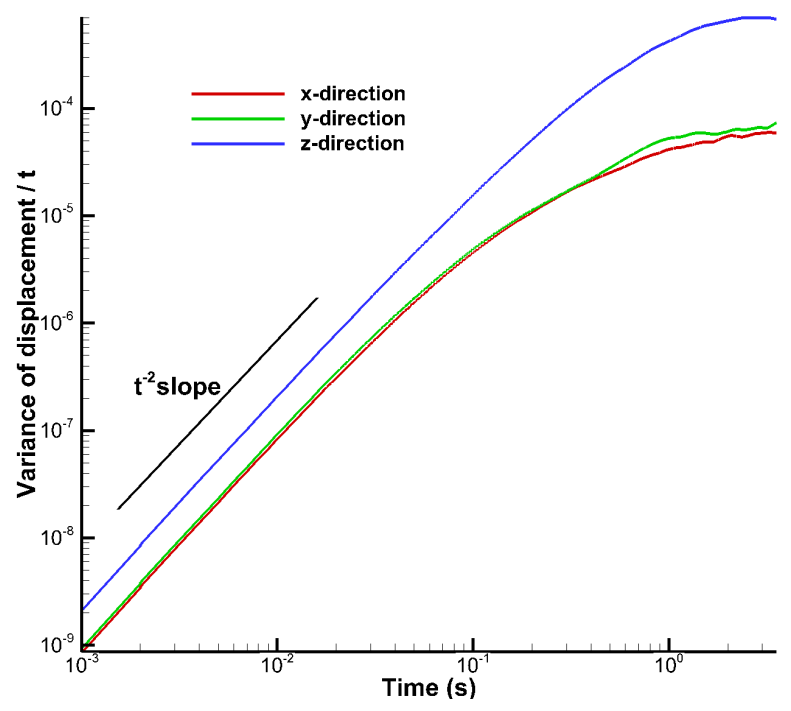

Figure 2: Variance of the displacement of the particles $\frac{M_{\alpha \alpha}}{t}$ according to time.

The first Lagrangian characteristics that can be studied are the particle velocity fluctuations over time in the fluidization regime, for $t>6 \mathrm{~s}$. In this particular flow conditions, the mean particle positions are null as the height of the bed height is constant over time. The numerically measured time averaged particle velocities are $\bar{U}_{x}=7.215010^{-5} \mathrm{~m} . \mathrm{s}^{-1}, \bar{U}_{y}=7.782210^{-5} \mathrm{~m} . \mathrm{s}^{-1}$ 
and $\bar{U}_{z}=1.412810^{-5} \mathrm{~m} . \mathrm{s}^{-1}$ respectively in the $x, y$ and $z$ directions. These values are four orders of magnitude smaller than the fluidization velocity and can be considered as zero.

\begin{tabular}{rrrr}
\hline & x-direction & y-direction & z-direction \\
\hline experiments of $[2]\left(m^{2} \cdot s^{-1}\right)$ & $5.4 \times 10^{-5}$ & $5.1 \times 10^{-5}$ & $6.8 \times 10^{-4}$ \\
simulations $\left(m^{2} \cdot s^{-1}\right)$ & $4.55 \times 10^{-5}$ & $5.5 \times 10^{-5}$ & $7.5 \times 10^{-4}$ \\
\hline
\end{tabular}

Table 1: Asymptotic values of $\frac{M_{\alpha \alpha}(t)}{t}$ for each Cartesian direction.

A last parameter of interest to feed the macroscopic Eulerian-Eulerian models devoted to fluidized beds is the autodiffusion coefficient $D_{\alpha \alpha}$. It is defined [2] according to the variance of the particle displacement $M_{\alpha \alpha}=<$ $\mathbf{X}_{i}-\mathbf{X}_{i, 0}>$ as

$$
D_{\alpha \alpha}=\lim _{t-t_{0} \rightarrow+\infty} \frac{M_{\alpha \alpha}(t)}{t}
$$

where $\mathbf{X}_{i}$ is the coordinate vector of one particle at a given time step and $\mathbf{X}_{i, 0}$ is the coordinate vector of the same particle at an initial time $t_{0}$. The notation $<\cdot>$ stands for the average over all the particles $i, i=1 . .2133$. In the experiments [2], it has been demonstrated that for small time steps, a $t^{-2}$ slope was observed for $D_{\alpha \alpha}$ whereas an asymptotic value was reached for larger times. This asymptotic value is the autodiffusion coefficient in a given direction. The curve of $\frac{M_{\alpha \alpha}(t)}{t}$ is presented in figure 2. The same slope as in the experiments in found numerically for small time steps. The asymptotic values obtained numerically and in the experiments for $D_{\alpha \alpha}$ are proposed in table 1. A good agreement is found between experiments and numerical solutions, the vertical component being one order of magnitude larger than in the horizontal directions. A $16 \%$ gap is observed between both approaches.

\section{Conclusion and perspectives}

A fictitious domain approach based on augmented Lagrangian techniques and penalty methods has been used for handling particulate flows of resolved scale particle. The work have been concentrated on the simulation of a real fluidized bed, in order to illustrate the ability of the ITPM to deal with dense particulate flows. The simulations have been favorably compared to reference experiments or theoretical results of the literature such as the fluidization velocity, variance of particle position or auto-diffusion coefficient. These simulations are a first step for managing numerical experiments with 
the DNS of particle flows. This is now possible on massively parallel computers, as 512 processors were used in the proposed simulations of fluidized beds.

\section{References}

[1] S. Vincent, J. C. Brändle de Motta, A. Sarthou, J.-L. Estivalezes, O. Simonin, and E. Climent. A Lagrangian VOF tensorial penalty method for the DNS of resolved particle-laden flows. submitted to Journal of computational Physics, pages 2523-2538, 2008.

[2] A. Aguilar Corona. Agitation of particles in a liquid fluidized bed. Experimental study. PhD thesis, Toulouse University, 2008.

[3] A. Ferrante and S. Elghobashi. On the physical mechanisms of two-way coupling in particle-laden isotropic turbulence. Physics of Fluids, 15:315-329, 2003.

[4] A M. Ahmed and S. Elghobashi. Direct numerical simulation of particle dispersion in homogeneous turbulent shear flows. Physics of Fluids, 13:3346-3364, 2001.

[5] P. Fede and O. Simonin. Numerical study of the subgrid fluid turbulence effects on the statistics of heavy colliding particles. Physics of Fluids, 18:045103045120, 2006.

[6] H. H. Hu, D. D. Joseph, and M. J. Crochet. Direct Simulation of Fluid Particle Motions. Theoretical and Computational Fluid Mechanics, 3:285-306, 1992.

[7] E. A. Fadlun, R. Verzicco, P. Orlandi, and J. Mohd-Yusofz. Combined Immersed-Boundary Finite-Difference Methods for Three-Dimensional Complex Flow Simulations. Journal of Computational Physics, 161:35-60, 2000.

[8] J. Kim, D. Kim, and H. Choi. An immersed-boundary finite-volume method for simulations of flow in complex geometries. Journal of Computational Physics, 171:132-150, 2001.

[9] S. Takagi, H.N. O guz, Z. Zhang, and A. Prosperetti. PHYSALIS: a new method for particle simulation Part II: two-dimensional Navier-Stokes flow around cylinders. Journal of Computational Physics, 187:371-390, 2003.

[10] M. Coquerelle and G.H. Cottet. A vortex level set method for the two-way coupling of an incompressible fluid with colliding rigid bodies. Journal of Computational Physics, 227:9121-9137, 2008.

[11] J. A. Simeonov and J. Calantoni. A pressure boundary integral method for direct fluid-particle simulations on Cartesian grids. Journal of Computational Physics, 230:1749-1765, 2011.

[12] K. Höfler and S. Schwarzer. Navier-Stokes simulation with constraint forces: Finite-difference method for particle-laden flows and complex geometries . Physical Review E, 61:7146-7160, 2000. 
[13] B. Maury. Direct simulations of 2D fluid-particle flows in biperiodic domains. Journal of Computational Physics, 156:325-351, 1999.

[14] R. Glowinski T. W. Pan, T. I. Hesla, D.D. Joseph, and J. Périaux. A fictitious domain approach to the direct numerical simulation of incompressible viscous flow past moving rigid bodies: application to particulate flow. Journal of Computational Physics, 169:363-426, 2001.

[15] Z. Zhang and A. Prosperetti. A second-order method for three-dimensional particle simulation. Journal of Computational Physics, 210:292-324, 2005.

[16] M. Uhlmann. An immersed boundary method with direct forcing for the simulation of particulate flows. Journal of Computational Physics, 209(2):448476, 2005 .

[17] M. Uhlmann. Interface-resolved direct numerical simulation of vertical particulate channel flow in the turbulent regime. Physics of Fluids, 20(5):053305, 2008.

[18] A. Ten Cate, J.J. Derksen, L.M. Portela, and E.A. Van Der Akker. Fully resolved simulations of colliding monodisperse spheres in forced isotropic turbulence. Journal of Fluid Mechanics, 519:233-271, 2004.

[19] H. Gao and L.-P. Wang. Lattice Boltzmann simulation of turbulent flow laden with finite-size particles. In Proceedings of the $7^{\text {th }}$ International Conference on Multiphase flows ICMF 2010, 2010.

[20] F. Lucci, A. Ferrante, and S. Elghobashi. Modulation of isotropic turbulence by particles of Taylor length-scale size. Journal of Fluid Mechanics, 650:5-55, 2010.

[21] F. Lucci, A. Ferrante, and S. Elghobashi. Is Stokes number an appropriate indicator for turbulence modulation by particles of Taylor-length-scale size? Physics of Fluids, 23:025101:1-7, 2011.

[22] J.-B. Ritz and J.P. Caltagirone. A numerical continuous model for the hydrodynamics of fluid particle systems. International Journal for Numerical Methods in Fluids, 30:1067-1090, 1999.

[23] T.N. Randrianarivelo, G. Pianet, S. Vincent, and J.-P. Caltagirone. Numerical modelling of the solid particle motion using a new penalty method. International Journal for Numerical Methods in Fluids, 47:1245-1251, 2005.

[24] T. N. Randrianarivelo, S. Vincent, O. Simonin, and J.-P. Caltagirone. A DNS approach dedicated to the analysis of fluidized beds. Fluid Mechanics Applications, 81:207-214, 2007.

[25] K. Khadra, Ph. Angot, S. Parneix, and J.P. Caltagirone. Fictitious domain approach for numerical modelling of Navier-Stokes equations. International Journal for Numerical Methods in Fluids, 34:651-684, 2000.

[26] J.F. Richardson and W.N. Zaki. Sedimentation and fluidization. Part 1. Transactions of the Institution of Chemical Engineering, 32:35-53, 1954. 